\title{
Bericht der österreichischen Rechtsexpertendelegation! über ihren Besuch in China/Tibet im Juli 1992
}

\author{
Von Felix Ermacora und Wolfgang Benedek
}

\section{Das Mandat, die Rahmenbedingungen und der Ablauf des Besuchs der öster- reichischen Rechtsexpertendelegation}

1. Anläßlich des Besuches des chinesischen Außenministers in Österreich ist mit dem österreichischen Bundesminister für Auswärtige Angelegenheiten die Entsendung einer Menschenrechtsexpertenkommission nach Tibet vereinbart worden. Ministerpräsident Li Peng meinte gegenüber der österreichischen Parlamentarierdelegation, die die Volksrepublik China im Jänner 1992 besuchte, daß eine österreichische Menschenrechtsexpertendelegation auch Peking besuchen solle. Er gehe davon aus, daß sich die Delegation an das von der Regierung vorbereitete Programm halten werde.

2. Der Bundesminister für Auswärtige Angelegenheiten und das BMfAA haben die Autoren dieses Beitrages ersucht, die oben genannte Besuchsreise vorzunehmen. Nach Klarstellung der Aufgabe und aufgrund geäußerter Wünsche kamen die Delegierten zu dem Schluß, sich als "österreichische Rechtsexpertendelegation" zu bezeichnen. Sie erhielten die Aufgabe, das Rechts- und Gesellschaftssystem Chinas, vor allem aber Tibets im Hinblick auf den Schutz und die Lage der Menschenrechte näher kennenzulemen und darüber dem BMfAA Bericht zu erstatten.

3. Die Erarbeitung des Besuchsprogrammes, in die im Wege des BMfAA die österreichische Botschaft in der Volksrepublik China eingeschaltet war, setzte im Februar 1992 ein. Kontakte mit zahlreichen hier nicht namentlich zu nennenden Experten, Wissenschaftlem, Politikern und von der chinesischen Politik Betroffenen wurden ebenso hergestellt wie eine Vorsprache beim Leiter der österreichischen Parlamentarierdelegation nach China, dem Präsidenten des österreichischen Nationalrates Univ.-Doz. Dr. H. Fischer vorgenommen. Letzterer und das BMfAA besorgten Berichte dieser Parlamentarierdelegation bzw. Berichte über den Chinabesuch anderer Delegationen (siehe auch Pkt. 9).

4. Die Beratungen über den Besuch bzw. das Besuchsprogramm fielen in den Zeitraum der 49. Tagung der Menschenrechtskommission der Vereinten Nationen, an der der Erstautor als Leiter der österreichischen Delegation teilgenommen hatte und vor allem die Beratungen über eine China- bzw. Tibetresolution - eingebracht von westlichen Delegationen - 
mitverfolgte. In diesem Zeitraum ist der UN-Menschenrechtskommission das UN-Dokument "Situation in Tibet, note by the Secretary General, submitted pursuant to Sub-Commission on Prevention of Discrimination and Protection of Minorities resolution 1991/ 10", E/CN. 4/1992/37 vorgelegen.

Eine entsprechende China/Tibet-Resolution westlicher Staaten, der sich auch Österreich als Sponsor aufgrund einer Weisung des BMfAA angeschlossen hatte, wurde nicht zur Abstimmung gebracht (siehe den Bericht über die 49. Tagung der Menschenrechtskommission im UN-Dok. E/1992/22 in Verbindung mit E/CN. 4/L. 49 Rev. 1).

5. Der Besuchtstermin für die österreichische Menschenrechtsdelegation, der zufolge anderer beruflicher Verpflichtungen der österreichischen Teilnehmer nicht unlimitiert war, wurde von österreichischer Seite zunächst für den April 1992 vorgesehen; dieser Termin wurde von chinesischer Seite nicht bestätigt; auch ein weiterer Terminvorschlag für den Monat September 1992 wurde nicht gutgeheißen, bis die chinesische Seite wissen ließ, daß ein Besuch in Tibet im Juli 1992 stattfinden könne. Berufliche Verpflichtungen erlaubten einen entsprechenden Besuch nur in der ersten Hälfte des Juli 1992. Der Termin 9. bis 19. Juli 1992 wurde schließlich gutgeheißen.

6. Das Besuchsprogramm, das von österreichischer Seite vorgeschlagen wurde und schließlich bei Ankunft in Peking am 10. Juli 1992 nach Gesprächen mit den einladenden Stellen und ein weiteres Mal am 13. Juli 1992 bei Ankunft in Lhasa noch einmal ergänzt wurde, entsprach weitgehend den österreichischen Wünschen. Ein Besuch in einem sogenannten Umerziehungs-Arbeitslager in Tibet konnte nicht erwirkt werden, wobei von chinesischer Seite nicht bestritten wurde, daß derartige Umerziehungslager existieren. Es war von österreichischer Seite auch nicht möglich gewesen, einen Tibetisch-Dolmetscher nach freier Wahl in die Delegation aufzunehmen. Solche Persönlichkeiten waren vorhanden und in Aussicht genommen. So mußte sich die österreichische Delegation mit Dolmetschern zufriedengeben, die von chinesischer Seite gestellt wurden. Dabei handelte es sich nicht um Berufsdolmetscher. Die Übersetzungen wurden von Angehörigen der österreichischen Botschaft in China, die der Delegation zugezogen wurden und wovon einer als ausgewiesener Sinologe die chinesische Sprache beherrscht, kritisch verfolgt, wobei das Bemühen des vom chinesischen Außenministerium beigegebenen Übersetzers, eines Diplomaten, um eine zutreffende Übersetzung bestätigt wurde.

7. Die Delegation setzte sich nach ihrer Konstituierung in Peking wie folgt zusammen: o. Univ.-Prof. F. Ermacora und Univ.-Doz. Dr. W. Benedek als Delegierte und die Herren BSR R. Hauser sowie Att. Dr. M. Allgäuer als fachkundige und chinaversierte Begleiter. Der österreichische Botschafter, bev. Min. Dr. Bukowsky betreute die Delegation in Peking und vermittelte die erforderliche Gesprächsatmosphäre mit den chinesischen Stellen in Peking. 
8. Wie im vereinbarten Besuchsprogramm vorgesehen, fanden in Peking Gespräche statt mit dem stellvertretenden Präsidenten des Chinese People's Institute of Foreign Affairs (CPIFA), das als die formell einladende Stelle auftrat, Herm Ma Xusheng, mit Herm Wang Jiafu, dem Direktor des Instituts der Rechtswissenschaften an der Akademie für. Sozialwissenschaften; mit Herm Wang Mingdi, dem stellvertretenden Direktor des Büros für AA im Ministerium für Justiz; mit dem Vizeaußenminister, Herm Yang Fuchang; mit Herm Li Gui, dem stellvertretenden Vorsitzenden des Minderheitenausschusses im Nationalen Volkskongreß; mit Herm Tudao Duoji, dem stellvertretenden Vorsitzenden der Minderheitenkommission im Staatsrat, Herm Zhu Entao, dem Leiter des Büros für auswärtige Angelegenheiten im Ministerium für öffentliche Sicherheit und Herm Chinai Luosang, dem stellvertretenden Vorsitzenden des staatlichen Amtes für Religionsangelegenheiten. An diesen Gesprächen nahm die chinesische Seite mit einer Reihe von Fachleuten und Fachbearbeitern der staatlichen Stellen teil.

In Lhasa fanden Gespräche mit dem stellvertretenden Vorsitzenden der Freundschaftsgesellschaft, Herrn Chen Bing, dem stellvertretenden Vorsitzenden der Kommission für Wirtschaftsplanung, Herm Geshang Wanjin, dem Prorektor der Universität Lhasa, dem stellvertretenden Direktor des Amtes für Rundfunk und Fernsehen, Herm Nima Zhandui, dem stellvertretenden Präsidenten des tibetischen VolksGH sowie Herm Chen Darong, Direktor des Amtes für Justiz, Frau Zhou Kaifu, stellvertretende Leiterin der Staatsanwaltschaft, Herm Yin Xingwen, dem Direktor des Gefängnisses Drapchi, einem Vertreter der Schulverwaltung und Herrn Suo Da, dem stellvertretenden Direktor der tibetischen Mittelschule in Lhasa, Herm Jampa Tiley, dem Leiter des tibetischen Krankenhauses und schließlich dem Vorsitzenden der autonomen Region Tibet, Herm Jiangcun Loubu, statt.

Die Delegation besuchte verschiedene religiöse Einrichtungen, so die Klöster Drepung und Ganden. Die Delegation hatte Gelegenheit, mit leitenden Mönchen in Ganden ein kurzes Gespräch zu führen. Die Delegation besuchte in Lhasa das berüchtigte Drapchi-Gefängnis, ohne jedoch - um Vorfälle wie in der Vergangenheit zu vermeiden - mit Häftlingen zu sprechen.

9. Die Delegation hatte den Eindruck, daß sie bei ihren Besuchen auf Schritt und Tritt beobachtet wurde und da $\beta$ die begleitende Seite tunlichst bestrebt war, ein nicht vorhergesehenes Gespräch, vor allem mit Mönchen, Gläubigen oder Tibetern, zu vermeiden. Die chinesische Begleitung war mit zurückhaltender Höflichkeit bestrebt, den volksrepublikanischen Standpunkt bei allen Belangen zu erklären und zu festigen.

Die Mitglieder der Delegation sind auf die Besuchsreise durch eine Fülle von allseitiger Literatur und menschliche Kontakte vorbereitet gewesen. Durch die eigene Anschauung ist sie auch im Stande, die chinesischen Strukturen der staatlichen Organisation in China und in Tibet besser zu verstehen und so - trotz der Kürze der Besuchszeit - ein abgewogenes Urteil über die gegenwärtige Lage der Menschenrechte in Tibet abzugeben. Das soll unter VI. zum Ausdruck gebracht werden. Sofort sei hervorgehoben, daß die privaten Menschenrechtsorganisationen, die außerhalb Chinas lebenden tibetischen Flüchtlinge, aber auch die 
oben genannte UN-Berichterstattung, die zwar kein eigenes Urteil abgibt, aber ihrerseits Berichte verwendet, einerseits, aber auch die chinesische Dokumentation andererseits ein Bild von Tibet und seiner Lage entwerfen, das nur von einer bestimmten Standpunkteinnahme in allen Details nachvollzogen werden kann. Dazu tritt schließlich, daß ausländische Delegierte mit ihrer Berichterstattung zum Bild des gegenwärtigen Tibet erheblich beitragen.

Die Delegation war im Besitze der Berichte der ausländischen Delegationen, die 1991/92 Tibet/China besucht haben. Es waren dies eine australische Menschenrechtsdelegation (Juli 1991), eine amerikanische Gruppe (August 1991), eine Delegation des Europäischen Parlaments (September 1991), eine Mission französischer Juristen nur nach China (Oktober 1991) sowie eine Schweizer diplomatische Delegation im Jahre 1992 mit Ausnahme von Besuchen nordischer und britischer Diplomaten aus Peking.

10. Die Menschenrechtslage im Tibet der Gegenwart ist nur verstehbar und verständlich, wenn man das Land vor seinem historisch-politischen Hintergrund einerseits und der Gesamtpolitik Chinas, vor allem der Menschenrechtspolitik andererseits sieht. Darauf sei im folgenden kurz eingegangen; wobei diese Darstellung weniger der eigenen Erfahrung als der literarischen Aufbereitung der Geschichte des eindrucksvollen Landes entspricht, die weitgehend standortbezogen ist.

\section{Zum historischen Aufriß der tibetanischen Frage}

11. Nach jahrhundertelangem politisch wechselhaften Geschick erklärte das Oberhaupt des Staates, der 13. Dalai Lama, im Jahre 1913 die Unabhängigkeit des Landes, eines Landes, das als Staat eine Monarchie mit theokratischer Regierungsform war, in der die Infrastruktur feudalherrschaftliche Züge aufwies, in denen das Mönchstum eine zentrale Stellung einnahm. Fast 3000 Klöster wies das dünnbesiedelte Land auf. Religiosität nach buddhistischen Glaubensgrundsätzen bestimmte nicht nur die Herrschaft, sondern auch die Leute. Die Religiosität der Tibeter ist auch heute noch, nach den Bedrückungen durch die kommunistische Herrschaft, vor allem durch die sogenannte Kulturrevolution und die Willkür der Roten Garden, unverkennbar: Sie ist das Land, sie ist die Kultur, sie ist die Kunst, sie ist die Lebensweise der Menschen in Stadt und Land. Das alles ist unverkennbar sichtbar. Der Besuch im "Haus des Herm", Jokhang, war für die Delegation besonders eindrucksvoll.

12. Die Kommunisten haben nach der Begründung der Volksrepublik China 1949 Anspruch auf Tibet erhoben. Taktische, politische und historische Gründe wurden für diesen Anspruch ins Treffen geführt, deren Richtigkeit hier dahingestellt bleiben darf. 1950 wurde Osttibet besetzt, am 15. November 1950 trat der 14. Dalai Lama als 15jähriger die Regierungsgeschäfte an. Am 23. Mai 1951 wurde unter politischem und militärischem Druck ein 17-Punkte-Plan zwischen China und dem Dalai Lama vereinbart. Mit diesem 
Vertrag wurde eine Art chinesisches Protektorat über Tibet errichtet. Nach der Zif. 4 dieses Vertrages - der Vertrag ist nicht im UNTS registriert - soll das bestehende politische System in Tibet unverändert bleiben, nach Zif. 4 sollen auch die Befugnisse und Funktionen des Dalai Lama nicht angetastet werden. Nach Zif. 7 sollen Religion, Sitten und Gebräuche des tibetischen Volkes respektiert und die Klöster geschützt werden.

Am 9. September 1951 marschierten die Soldaten der Volksbefreiungsarmee Chinas - wie zeitgenössische Photographien zeigen - mit klingendem Spiel, aber ohne Volksbeteiligung in Lhasa ein. Eine Annexion wurde vollzogen. Ob das Abkommen mit dem 14. Dalai Lama völkerrechtsgemäß war, sei hier dahingestellt.

Ähnlich wie in Afghanistan im Jahre 1978/79 löste die chinesische Kulturpolitik, die einem "Husarenritt" glich, Volkswiderstand aus, dessen die übermächtige chinesische Gewalt jedoch bald Herr wurde. Die Internationale Juristenkommission berichtet, daß zwischen 1951 und 1959 mehr als 90.000 Tibeter im Widerstand gegen die chinesischen Besatzer umgekommen sind. 1959 flüchtete der Dalai Lama nach Indien, 80.000 Tibeter gingen mit ihm ins Exil, wo der Dalai Lama eine Exilregierung begründete.

Im Zuge der heute allseits verurteilten Kulturrevolution sind von 1966 bis 1976 von den einst fast 3000 Klöstem des Landes nur etwa 20 erhalten geblieben. Die Expertengruppe konnte die berühmten Klöster Ganden und Drepung besuchen. Das erstgenannte wurde geradezu vollkommen zerstört und ist - wie die Mönche sagten - auch von Landsmännem ausgeraubt worden, Drepung blieb in seiner Pracht weitgehend verschont, auch Sera.

Der Annexionswille Chinas kommt in der Errichtung einer Autonomen Region Tibet ("Xizang") im Jahre 1965 deutlich zum Ausdruck.

13. Ab 1980 wird zwar in einer Liberalisierungspolitik Tibet an eine längere Leine genommen, was aber in den Jahren 1987, 1988 und 1989 Aufstände gegen die als Besatzungsregime empfundene chinesische Herrschaft nicht verhindern konnte.

Der Dalai Lama alarmiert die westliche Welt, er stellt im Jahre 1987 vor dem US-Repräsentantenhaus ein Forderungsprogramm auf, das im wesentlichen auf die Erhaltung der tibetischen Identität gerichtet ist. Im Jahr 1989 erhält der Dalai Lama den Friedensnobelpreis. Menschenrechtliche Kritik wird im Westen und in den UN laut.

Die Haltung des Dalai Lama zu Tibet beunruhigt die chinesischen Gesprächspartner. Der Dalai Lama hat im Jahre 1991 seinen Fünf-Punkte-Plan zurückgezogen, in dem er sich of fensichtlich mit einer starken Autonomie zuf riedengegeben hatte. So erklärte der Vorsitzende der autonomen Region (Jiangcun Loubu) in einem Gespräch mit der österreichischen Delegation, in dem er gefragt wurde, ob man nun die Probleme der Jahre 1986 bis 1989 gelöst habe, u.a. wörtlich:

"Ich möchte sagen, daß diese Auseinandersetzungen um die Einheit Tibets mit China oder Separatismus fortgehen wird. Diese gab es in der Vergangenheit, in der Gegenwart und wird es in der Zukunft geben. Der Grund liegt darin, daß der Dalai Lama nach seiner Flucht das Vaterland verraten hat und separatistische Aktivitäten entwickelt. ... 
Peking sagt, wenn der Dalai Lama seine Unabhängigkeitsziele aufgibt, dann kann man über alles sprechen. Tibet wird sein Tor nicht zuschlagen. Ob es zur Ruhe kommen wird - ich glaube, wenn sich Gelegenheit bietet, werden die Separatisten weiter ihre Sache tun.

Ich kann mit meiner Autorität sagen, daß die Unabhängigkeit Tibets unmöglich ist, weil die Bevölkerung Tibets damit nicht einverstanden ist. Die Lösung der Tibetfrage liegt ausschließlich darin, daß der Dalai Lama seine Zielsetzungen auf gibt."

(Siehe zur Politik des Dalai Lama neuerdings die Ausführungen des äußerst versierten W. V. Erffa, "Warten, bis es in China eine neue Situation gibt. Tibet und der Dalai Lama geben die Hoffnung auf Befreiung nicht auf", FAZ v. 7.2.1992, Nr. 32, S. 8).

14. Die Autonomie "Kleintibets" im chinesischen Staatsverband als eine autonome Region (9.9.1965) kann nicht verbergen, daß ein allfälliger Autonomiekonflikt, wie man ihn in den österreichisch-italienischen Beziehungen betreffend Südtirol erlebt hat, dann nicht die Sache trifft, wenn man vom transzendenten Bezug des Problems absieht. Der Konflikt um Tibet ist ein Wertekonflikt, in dem eine marxistisch-maoistisch-materialistische Idee einer mit traditionellen Werten, gleichgültig wie man diese auch charakterisieren mag, verhafteten Bevölkerung gegenübersteht. Wenn man, wie es scheint, die religiösen Freiheiten wiederum herstellt, so ist auf Dauer gesehen der Wertekonflikt nur so zu lösen, daß an die Stelle des tibetischen Menschen ein moderner industrie- und technikbezogener Mensch tritt. Volksgruppenmäßig gesehen heißt dies, daß nur "Volkstod durch Unterwanderung" das Tibetproblem im chinesischen Sinne wird lösen können. Das aber ist ein Problem, das in den modernen Volksgruppen- und Minderheitenschutz hineinreicht, zu dem die chinesische Menschenrechtspolitik das ihre beizutragen hat (siehe IV.).

15. Dic Expertendelegation hat sich bemüht, die Strukturen der autonomen Provinz Tibet zu ermitteln. Um sie zu verstehen, ist zu allererst zu sagen, daß sie, wie alle übrigen staatsrechtlichen Strukturen vom klassischen Prinzip kommunistischer Staatsführung, dem Prinzip des demokratischen Zentralismus, beherrscht ist. Das gilt auch für die dezentralen autonomen Ausgliederungen. Sie werden von der KPCh beherrscht. Einheit der Staatsgewalt bedeutet Einheit des Parteiwillens. Wenn die gegenwärtige wirtschaftliche Öffnung diesem Prinzip des demokratischen Zentralismus nicht mehr Hilfe sein kann, so ist dies zunächst ein marxistisch-dogmatisches Problem, das in diesem Bericht auf sich beruhen bleiben kann.

Die tibetanische Region hat an autonomen Strukturen einen Volkskongreß und eine Volksregierung mit tibetischen Vorsitzenden sowie eine Verwaltungsstruktur, die sowohl Aufgaben der autonomen als auch der lokalen Verwaltung wahmimmt. Diese Strukturen sind durch Art. $112 \mathrm{ff}$. der Chinesischen Verfassung von 1982 vorgegeben und durch Gesetze, wie insbesondere das Gesetz über die regionale Autonomie von 1984, näher ausgeführt. Hinsichtlich der Modernisierung des Gesellschaftswesens, insbesondere die technische 
Durchdringung und Öfnung des Landes und den Versuch, die Tibeter in diese Modemisierung zu integrieren, wird die chinesische Leistung zu beachten sein. Ob sie jedoch im Stande ist, das transzendente tibetische Wertsystem auszumerzen, ist eine andere Frage.

\section{Die menschenrechtlichen Aspekte der tibetischen Frage - Allgemeines}

16. Die chinesische Besetzung Tibets und die behaupteten Menschenrechtsverletzungen gegenüber dem tibetischen Volk und seinen Angehörigen hat die Generalversammlung der Vereinten Nationen in mehreren Resolutionen veranlaßt, auf die Beachtung des Selbstbestimmungsrechtes des tibetischen Volkes und die Achtung der Menschenrechte, vor allem das Recht der Religionsfreiheit, hinzuweisen (siehe die Res. 1353 [XIV], 1723 [XV] und $2079[X X])$.

17. Die blutige Niederschlagung der Aufstände der Tibeter in den Jahren 1987, 1988 und 1989 haben das Europäische Parlament und die Parlamentarische Versammlung des Europarates veranlaßt, gegen die Unterdrückung der tibetischen Religion und Kultur Stellung zu nehmen, aber zugleich die allmählich einsetzende Politik der Toleranz und die Bedeutung des 5-Punkte-Planes des Dalai Lama, den er auch in Straßburg vorgelegt hatte, hervorzuheben (EG-Parlament vom 14.10.1987). Die Sorge um den Verlust der tibetischen Identität ist zum Ausdruck gebracht worden (Parlamentarische Versammlung des Europarates in der "Written Declaration" Nr. 173 v. 5.10.1988). Von seiten des EG-Parlamentes sind die blutigen Ereignisse in Tibet vom März 1989 kritisch beurteilt worden (siehe Entschließung vom 15.3.1989).

18. Die Vereinten Nationen haben sich des weiteren zunächst aufgrund eines Verlangens der Unterkommission zur Beseitigung der Diskriminierung und zum Schutze der Minderheiten vom 23.8.1991 (Res. 1991/10) unter Berufung auf Berichte über die lauf ende Verletzung von Menschenrechten, die die hervorragenden Merkmale kultureller, religiöser und nationaler Identität des tibetischen Volkes gefährden, mit der Situation in Tibet befaßt. Der Generalsekretär hat der Menschenrechtskommission der UN - wie schon hervorgehoben eine Note über diese Situation vom 5.2.1992 zugemittelt. Sie enthält eine Stellungnahme der Volksrepublik China zum genannten Fragenkomplex, Stellungnahmen von NGOs und Presseauszüge. Die Berichte des Spezialberichterstatters der Menschenrechtskommission der UN über willkürliche Exekutionen (siehe UN-Dok. E/CN. 4/1991/30), des Spezialberichterstatters über die Folter (siehe UN-Dok. E/CN. 4/1992/17), der Arbeitsgruppe über die verschwundenen Personen (siehe UN-Dok. E/CN. 4/1992/18) und des Spezialberichterstatters über die religiöse Intoleranz (siehe UN-Dok. E/CN. 4/1992/52) enthalten gleichfalls Verweise auf Berichte von Menschenrechtsverletzungen in den verschiedenen Lebensbereichen des tibetischen Volkes. Wie schon ausgeführl, konnte aber eine Resolu- 
tion westlicher Staaten zum Menschenrechtskomplex der Volksrepublik China/Tibet in der Menschenrechtskommission der UN 1992 nicht zur Abstimmung gebracht werden.

19. Die Regierung der Volksrepublik China hat offenbar unter dem Druck dieser internationalen Befassungen mit der Menschenrechtsf rage in Tibet einerseits und zur Bekundung der Transparenz in dieser Frage, ohne ihren grundlegenden Menschenrechtsstandpunkt (siehe Pkt. 20) zu revidieren und offenbar um unter Beweis zu stellen, daß es in Tibet eine Politik der Toleranz gebe - z.B. hinsichtlich des Wiederaufbaus zerstörter tibetischer Klöster, der Teilnahme der Tibeter an der autonomen Verwaltung der Region Tibet - andererseits, politischen und Expertenkommissionen gestattet, China und Tibet zu bereisen.

Die österreichische Expertendelegation war innerhalb relativ kurzer Zeit die fünfte dieser Art, die Tibet besucht hat (vgl. Pkt. 9).

20. Die Volksrepublik China hat in einem Grundsatzpapier "Human Rights in China. Information Office of the State Council", November 1991, Beijing, China, 58 Seiten (Text auch in deutscher Sprache), ihren Standpunkt zur Menschenrechtsfrage im allgemeinen und zur Tibetfrage im besonderen öffentlich gemacht (seit Abfassung dieses Berichts wurden von China zwei weitere Grundsatzpapiere über "Criminal Reform in China" [August 1992] und "Tibet - Its Ownership and Human Rights Situation" [September 1992] veröffentlicht). Dieser Standpunkt ist auch in den Foren der UN und gegenüber der österreichischen Expertendelegation mündlich wiederholt worden. Die chinesische Seite verschloß sich einer grundlegenden Diskussion ihrer Haltung nicht bzw. nahm einen anderen Standpunkt zur Kenntnis, der zu dieser Frage von österreichischer Seite vorgebracht worden ist.

Die Position der Volksrepublik China zur Menschenrechtsfrage im allgemeinen läßt sich aufgrund der Dokumentation und der gefuhrten Gespräche wie folgt zusammenfassen:

a) Vorrang des Rechtes auf Lebensunterhalt und Existenzminimum (right to subsistence) und das Recht auf Verbesserung der Lebensbedingungen (right to development);

b) unter dieser Voraussetzung Vorrang der Rechte der Kollektivität und der Abschirmung in ideologischer und politischer Hinsicht, aber auch hinsichtlich der Entfaltung der Persönlichkeit, soweit dies vor allem die Familienplanung angeht,

c) Recht auf Privateigentum, soweit dieses die wirtschaftliche Entwicklung fördern kann und am Grundsatz des öffentlichen Eigentums als Baustein der chinesischen Wirtschaftspolitik nicht rührt;

d) in den internationalen Bezichungen, die menschenrechtliche Fragen berühren, bei grundsätzlicher Anerkennung der Universalität der Menschenrechte, strikte Beachtung der domestic jurisdiction clause (Art. 2 Zif. 7 der UN-Charta), d.i. der Souveränität. Das bedeutet, daß sich die Volksrepublik China gegen bestimmte Rechtsauffassungen von dritten Staaten oder von Internationalen Organisationen verwehrt. Hier beruft sich die Volksrepublik China auf die klassische "peaceful coexistence clause" der BandungKonferenz (1955). 
21. Die Betonung der Bedeutung der domestic jurisdiction-Klausel in den internationalen Beziehungen ist ein wichtiger Streitpunkt bei der Beurteilung der Tibetfrage, weil die strikte Anwendung der genannten Klausel von west- und osteuropäischer Seite heute erheblichen dynamischen Einflüssen unterworfen ist. China betont, daß es von den derzeit 25 menschenrechtlichen Konventionen der UN acht beigetreten ist, unter anderem der Antisklavereikonvention, der Internationalen Konvention über die Bekämpfung und Bestrafung des Verbrechens der Apartheid, der Konvention über die Beseitigung aller Formen rassischer Diskriminierung, der Konvention über die Beseitigung aller Formen der Diskriminierung der Frau, der Konvention gegen den Völkermord und der Konvention über das Verbot der Folter.

Im Gespräch mit dem Vize-Außenminister der Volksrepublik China führte dieser zur Haltung Chinas in intemationalen Menschenrechtsfragen u.a. folgendes aus:

"... Wenn China chaotisch würde wie die osteuropäischen Staaten und die ehemalige UdSSR, würde es noch schwierigere Probleme geben. Was die Menschenrechte angeht, so legen wir Wert darauf, die Menschenrechte zu beachten. Im Rahmen der Gesetze genießen die Bürger Chinas umfassende Rechte, das wisse man ganz genau. Der Vergleich zwischen der Vergangenheit und der Gegenwart mache dies deutlich. ... Die Bevölkerung schätzt die gegenwärtige Situation sehr hoch ein. ... Wir sind natürlich gegen jegliche Aktivitäten, die für einen Umsturz eintreten.

... Wir sind der Meinung, daß die Menschenrechtsfragen der Innenpolitik zugehören. Ein Land soll in den Menschenrechtsfragen von den konkreten Gegebenheiten ausgehen und selbst entscheiden, wie die Probleme zu lösen sind. Wenn Probleme auftauchen, und sich die Länder einmischen, wird es zu einem Durcheinander kommen.

... Bei der Betrachtung eines Menschenrechtszustandes kann man nicht von der Geschichte des Landes losgelöst vorgehen. Viele Berichte der westlichen Journalisten entsprechen nicht der Realität. Das ist nicht die Schlußfolgerung von mir, sondern von vielen Fremden aus Europa..."

Auf eine Frage bezüglich des Dalai Lama erklärte der chinesische Politiker: "Unsere Haltung hat sich nicht geändert. Der Dalai Lama hat einen Dialog vorgeschlagen. Außer der Frage über die Unabhängigkeit Tibets können wir über alle Fragen mit ihm diskutieren. Unsere generelle Haltung ist der Dialog, aber nicht über die Unabhängigkeit Tibets. Doch der Dalai Lama hat die Tür zum Dialog selbst zugeschlagen." Der Vize-Außenminister verweist auf seinen Besuch in Indien. Indien habe zwei Standpunkte begründet: "Indien anerkennt Tibet als einen Teil Chinas und Indien erlaubt keine antichinesische Aktivität in Indien. Nepal hat einen ähnlichen Standpunkt bezogen..."

Die Ratifizierung der Menschenrechtspakte der UN sei Gegenstand einer Prüfung, meinte der Politiker.

22. Wenn man die internationalen Dokumente und die staatlichen Berichte zur Menschenrechtsfrage in China und in Tibet im besonderen überblickt und die Beobachtungen der 
österreichischen Expertendelegation in Rechnung stellt, so ergeben sich für die Tibetfrage vom menschenrechtlichen Gesichtspunkt folgende Hauptprobleme:

- die Frage nach der Entidentifizierung Tibets durch Gleichschaltung und den Versuch der Unterwanderung des tibetischenVolkes,

- die Bedeutung der Autonomie Tibets im chinesischen Staatsverband als Mittel der Gleichschaltung oder als Garantie der Erhaltung tibetischer Identität,

- die sich aus diesen Fragen und ihren Beantwortungen ergebenden Auswirkungen auf den Umfang der wirtschaftlichen, sozialen und kulturellen Rechte sowie der bürgerlichen und politischen Rechte der Tibeter. Gerade diese Frage ist einer weiten Ausfächerung zugänglich, der die Expertendelegation im einzelnen nachgegangen ist.

Die Fragen werden unter Bedachtnahme auf das Wissen um die erschreckenden Ereignisse der chinesischen Kulturrevolution und um die Unterdrückung der Aufstände der Jahre 1987, 1988 und 1989 sowie die Art und Weise dieser Unterdrückung zu beantworten sein. Zugleich ist zu bedenken, daß sich die chinesischen Autoritäten hinsichtlich aller kritischen Fragen - von der Anerkennung des Fehlverhaltens während der Kulturrevolution abgesehen - ungemein bedeckt geäußert haben, daß Kontakte in Tibet mit offen sprechenden Tibetern nahezu ausgeschlossen waren und man daher zwischen dem, was man an Ort und Stelle hören und sehen kann, und dem, was man aus anderen, vor allem ausländischen Quellen zu hören bekommt, zu unterscheiden hat. Im Nachstehenden ist auf die einzelnen Fragen vor allem unter Bedachtnahme auf die weitverbreiteten Vorwürfe einzugehen.

\section{Unabhängigkeit Tibets und Nationalitäten-Minderheiten}

23. Die Vorwürfe, die der Volksrepublik China seit der "friedlichen Befreiung" Tibets, d.i. die militärische Besetzung Tibets im Jahre 1950/51, gemacht werden, beziehen sich auf die Mißachtung des Selbstbestimmungsrechts des tibetischenVolkes und auf die dadurch bewirkte Entfremdung Tibets von seinen religiösen, sprachlichen und ethnischen Charakteristiken. Die für Tibet heute eingerichtete regionale Autonomie (TAR) und der Minderheitenschutz in China bieten demgegenüber - wie noch hervorgehoben wird - keinen gleichwertigen Ersatz.

24. Das Selbstbestimmungsrecht der Völker steht heute allen Völkem zu. Die Stimmen, die seine Ausübung nur als ein Mittel des Entkolonisierungsprozesses sehen wollen, sind bei weitem in der Minderheit. Das Selbstbestimmungsrecht gehört heute zu jenen Rechten, die von den Vereinten Nationen und der Völkergemeinschaft als zwingendes Recht (ius cogens) angesehen werden, das Verträge derogiert, die entgegen dem Selbstbestimmungsrecht abgeschlossen worden sind. Die Generalversammlung der Vereinten Nationen hat in ihrer Resolution 2625 (XXV) die wesentlichen Modelle der Selbstbestimmung bezeichnet. Es steht den Völkern zu, das jeweilige Modell der Selbstbestimmung in freier Entscheidung zu bestimmen. Zum Recht auf Selbstbestimmung gehört auch das Recht der betroffenen 
Völker, über die dem Lande zugehörigen wirtschaftlichen Güter frei zu verfügen. Das Selbstbestimmungsrecht verpflichtet die betroffenen Staaten, ein Verfahren festzulegen, kraft dessen Selbstbestimmung friedlich ausgeübt werden kann. Das von den Vereinten Nationen proklamierte Selbstbestimmungsrecht findet seine Grenze in der Integrität eines gegebenen staatlichen Territoriums. Das bedeutet aber nicht, daß über das Selbstbestimmungsrecht und seine Ausübung im konkreten Fall nicht verhandelt werden könnte.

25. Im Hinblick auf die historische, religiöse und demographische Entwicklung Tibets steht außer Zweifel, daß die ursprüngliche Bevölkerung Tibets - ohne Bedachtnahme auf die chinesischen Zuwanderer - als Volk gelten kann. Die Tibeter haben alle Charakteristika eines Volkes, wie diese von Volkstums-Wissenschaft und Praxis gesehen werden: das angestammte Territorium, wozu heute zumindest das Gebiet der autonomen Region Tibet gehört; die eigene Sprache, die mit dem Chinesischen so wenig zu tun hat wie das Deutsche mit dem Italienischen und eine lebende Sprache ist; die den Tibetern eigene Religion - die des tibetischen Buddhismus -, die die besondere Charakteristik des tibetischen Volkes kennzeichnet; die aus der Religion folgenden kulturellen Charakteristika, die sich über Jahrhunderte hinweg bis in die Gegenwart erhalten haben; und der unbestreitbare Wille der Mehrheit der Tibeter, Religion, Sprache und kulturelle Charakteristika zu bewahren.

26. Man hat den Eindruck, daß eine langfristig ins Auge gefaßte demographische Veränderung des tibetischen Volkes durch die Volksrepublik China im Gang ist, die sicherstellen soll, daß die derzeitige nationale Einheit Chinas nicht gefährdet werde. Davon zeugen die starke Präsenz der chinesischen Volksbefreiungsarmee (PLA), vor allem im Raum um Lhasa, und die bevorstehende Verlagerung des Hauptquartiers der PLA für die autonome Region Tibet (TAR) von Chengdu nach Lhasa; die Präsenz einer chinesischen Beamtenschaft bzw. von in China ausgebildeten tibetischen Beamtenkadern; die dem kommunistischen Staatssystem entsprechend ausgerichteten Parteikader, die, mögen ihre Mitglieder auch Tibeter sein, im Wertekonflikt zwischen dem Dalaiismus und dem Maoismus auf der Seite des Maoismus stehen. Dazu kommen die Familienangehörigen der Mitglieder der militärischen und sicherheitspolizeilichen Beamten- und Parteikader, die alle samt und sonders das öffentliche Leben in Tibet zumindest in den Ballungsräumen beherrschen. Dazu zählen ca. 100.000 Han-Chinesen. Nicht erfaßt sind darin die nicht ständig in Tibet lebenden Lehrer, Experten etc.

Auch wenn von chinesischer Seite die Chinesisierung Tibets als politisches Ziel bestritten wird, so ist sie aus einer Reihe von Einzelheiten als Faktum erkennbar: Im öffentlichen Sprachgebrauch wird tunlichst vermieden, zwischen Chinesen und Tibetem zu unterscheiden, wenn, dann wird von tibetischen und $\mathrm{Han}$-Chinesen gesprochen; im Erziehungssystem wird die tibetische Intelligenz in der Regel außerhalb Tibets in China ausgebildet; Lizenzen für Gewerbe und Industrie werden vor allem Chinesen zuerkannt, dazu zählen ca. 300.000 Chinesen, die als "floating population" eingeschätzt werden und vor allem in den beiden Ballungsräumen siedeln, oder es wird danach getrachtet, unter Berufung auf den wirt- 
schaftlichen Fortschritt in Tibet die Infrastruktur von Handel, Gewerbe und Industrie nach chinesischen Gesichtspunkten zu ordnen. Selbst in den Klöstern sind seit der Kulturrevolution "demokratische Verwaltungskomitees" aufgebaut, die auch eine Art politische Kontrollfunktion innerhalb der klösterlichen Gemeinschaften ausüben. Der österreichischen Expertendelegation ist bekanntgeworden, da $\beta$ die Klöster große Schwierigkeiten haben, qualifizierte Lehrer für die Ausbildung der Mönche zu finden.

Es mag sein, da $\beta$ all dem das Argument entgegengehalten wird, da $ß$ mit solchen Methoden der Fortschritt am "Dach der Welt" einziehen werde. Aber von der Hand gewiesen können die eben angestellten Uberlegungen und Beobachtungen nicht werden. Allerdings ist die chinesische Bevölkerungsplanung für die Tibeter günstiger: Die Han-Chinesen-Familien sind auf die Ein-Kind-Familie vorprogrammiert, tibetischen Familien werden je nach ihrem Status zwei, drei oder mehr Kinder erlaubt. Demgegenüber berichten Exiltibeter von Zwangssterilisation an tibetischen Frauen in chinesischen Kliniken Tibets oder von Zwangsabtreibungen in bezug auf Familien tibetischer Kader. Derartigen Berichten wird in Tibet allerdings widersprochen.

Endlich wolle nicht übersehen werden, daß die "friedliche Befreiung" Tibets, die Willkür der Kulturrevolution und die Wirkungen der Niederschlagung der Aufstände 1987, 1988 und 1989 Zigtausende von Tibetem gezwungen und veranlaßt haben, ihre Heimat zu verlassen und als Flüchtlinge ihr Heil in anderen Regionen der Welt zu suchen. Die Zahl der tibetischen Exilanten geht in die Hunderttausende. Auch die Flüchtlingswellen tragen zur demographischen Veränderung eines Gebietes bei. Jedenfalls besteht die Elite in Tibet entweder aus Han-Chinesen oder ist weitgehend chinesisch assimiliert und kontrolliert.

27. Der Blick auf die intemationalen Dokumente zur Tibetfrage - Resolutionen von Organen der Vereinten Nationen, der EG und des Europarates - zeigt, da $\beta$ von ihnen entweder die Gewährung des Selbstbestimmungsrechtes für das tibetische Volk ausdrücklich verlangt wird oder die Achtung der Menschenrechte schlechthin, zu denen zweif elsohne auch das Selbstbestimmungsrecht der Völker gehört, verlangt wird.

Die chinesische Verfassung (siche die Ausgabe in der Sammlung Blaustein/Flanz, Constitutions of the World, Peoples Republic of China) und das chinesische Weißbuch für Menschenrechte enthalten kein Bekenntnis zum Selbstbestimmungsrecht, vom Selbstbestimmungsrecht der unter Kolonialherrschaft stehenden Völker abgesehen. Angesprochen auf Tibet, erklärten alle chinesischen Gesprächspartner, daß eine "Unabhängigkeit Tibets" nicht in Frage komme. Die Anwendung dicses Modells der Selbstbestimmung der Tibeter wird damit von chinesischer Seite von vornherein ausgeschlossen. Das ist nicht nur Theorie, sondern mündet in das tägliche Leben: Ein Gebet, in dem von der Unabhängigkeit Tibets die Rede ist, darf weder in Klöstem noch in der Öfentlichkeit auf den Pilgerpfaden gesprochen werden. Tibetische Flüchtlinge, die wegen dieses Gebetes in Haft gewesen sind, haben bestätigt, daß sie wegen der Forderung nach der Unabhängigkeit Tibets gemäß den straf rechtlichen Bestimmungen wegen kontrarevolutionärer Handlungen verfolgt worden sind. 
Im Zuge des wirtschaftlichen Fortschrittes werden die natürlichen Ressourcen Tibets von China genützt; die Wälder abgeholzt, Uranium und andere Mineralien für die chinesische Atomenergie genützt, Entwicklungsprojekte wie die Nutzung der Wasserwirtschaft, der Straßenbau, der Bau von Flughäfen kommen in erster Linie China und chinesischen Einwanderem zugute. Der wirtschaftliche Fortschritt steht in offenem Gegensatz zu den hergebrachten Vorstellungen der Tibeter, die sie über die Nutzung von Grund und Boden haben.

28. Demgegenüber wurde Tibet unter schriftlicher und mündlicher Betonung der Einheit Chinas als Staatsganzes und des demokratischen Sozialismus eine regionale Autonomie im Rahmen des chinesischen Staatsverbandes zuerkannt. Die autonome Region Tibet umfaßt 75 Bezirke und zwei Städte - Lhasa und Xigaze. Neben der autonomen Region Tibet gibt es 10 autonome Präfekturen und zwei autonome tibetische Bezirke in China. Es handelt sich bei der Autonomie nicht um eine Sonderautonomie, wie man sie etwa im italienischen Staatsverband für Südtirol kennt. Gemäß den Art. 112 bis 122 der chinesischen Verfassung gibt es nur ein Autonomiemodell. Es kommt autonomen Regionen, autonomen Präfekturen und Bezirken zu. Neben den Grundzügen der autonomen Strukturen, die in die Staatsverfassung aufgenommen sind, gibt es ein Gesetz aus dem Jahre 1984, das sich speziell mit der regionalen nationalen Autonomic befaßt: "Law of the Peoples Republic of China on Regional National Autonomy" vom 31. Mai 1984. Dieses Gesetz enthält 67 Artikel, ist aber nicht speziell auf Tibet zugeschnitten, sondern auf alle autonomen Gebilde.

Aus diesen Regeln ist zu entnehmen, da $\beta$ die regionale Autonomie eine Art Gesetzgebungsund Verwaltungsautonomie umfaßt. Im Art. 119 der Verfassung werden Fragen der Erziehung, der Wissenschaft, der Kultur, des Gesundheitswesens hervorgehoben. Gemäß Art. 120 der chinesischen Verfassung wird den Selbstverwaltungsorganen die Kompetenz zuerkannt, Kräfte örtlicher Sicherheitspolizei für die Aufrechterhaltung der öffentlichen Ordnung einzurichten. Art. 121 der Verfassung garantiert eine gewisse Sprachautonomie. Gemäß Art. 122 der Verfassung werden die finanziellen Beziehungen zwischen dem Staat und den autonomen Regionen geregelt: Der Staat gibt den nationalen Minderheiten finanzielle und technische Assistenz. Er bildet für die nationalen autonomen Gebiete in großer Zahl die Kader der Bediensteten auf allen Ebenen der lokalen Verwaltung aus. Das Menschenrechtsweißbuch Chinas gibt nähere Details über diese Kaderausbildung: "Now there are 37.000 Tibetan cadres throughout Tibet, making up $66,6 \%$ of the total number of cadres; this breaks down to about 72 percent at autonomous region level and $61,2 \%$ at county level..."

29. Die Gerichtsbarkeit und das Justizwesen fallen nicht in den autonomen Bereich.

30. Aus dem oben genannten Autonomiegesetz geht die Zielsetzung der Autonomie weitaus klarer hervor als aus der Verfassung Chinas selbst. Der Rahmen der Autonomie ist die Stärkung der nationalen Einheit Chinas und der Aufbau der sozialistischen Modernisierung. Dazu gehört einerseits die Bctonung, daß alle nationalen autonomen Gebiete integrale Teile 
der chinesischen Republik sind und daß auch die autonomen Organe dem Prinzip des demokratischen Zentralismus folgen sollen (Art. 3). Die Sabotage der nationalen Einheit und Verabredungen über nationalen Separatismus sind verboten (Art. 9). Eine ähnliche Schranke ist religiösen Aktivitäten auferlegt (Art. 11). Die Organisation der Autonomie ist kommunistischen Organisationsmethoden unterworfen. Das drückt sich vor allem im Prinzip der sogenannten doppelten Unterstellung aus, dem die Verwaltungsorgane unterworfen sind: "The people's government in national autonomous areas shall be responsible and report its work to the people's representative congress at the same level and the higher State administrative organs one level above it." (Art. 15)

In der Verwaltungsorganisation soll eine Art Ausgleich zwischen der Repräsentation der Nationalitäten, die in einem autonomen Gebiet siedeln, erfolgen. Auch das entspricht einem gewissen verwaltungsinternen Kontrollmechanismus und macht verständlich, warum die Volksrepublik China bestrebt ist, nicht-tibetische Chinesen in Tibet wohnhaft und seßhaft zu machen. Neben dem Vorsitzenden, der aus der autonomen Region zu stammen hat, haben andere Verwaltungsorgane anderen Nationalitäten entnommen zu werden (Art. 17).

Das Autonomiegesetz von 1984 enthält eingehende Regelungen über den Gebrauch der Lokalsprache und das Erziehungssystem sowie über die Infrastruktur der Organisation der freien Meinungsäußerung (Art. 37 und 38 ff.).

Die Gerichtsbarkeit in den autonomen Regionen ist zentralistisch orientiert. In bezug auf den wirtschaftlichen Fortschritt ist eine enge Zusammenarbeit zwischen Organen der autonomen Region und jenen des Zentralstaates vorgesehen.

Die Gespräche mit Vertretern öffentlicher Einrichtungen in Tibet haben die Anwendung all der eben genannten Grundsätze in der autonomen Region Tibet deutlich gemacht. Sie machten auch sichtbar, da $\beta$ die Zentralregierung den wirtschaftlichen und sozialen Fortschritt unter Berufung auf die Rückständigkeit des Landes vor allem auf die Zusammenarbeit der lokalen und zentralen Dienststellen gründet. Die Gesprächspartner wiesen auch auf das Bestehen einer Durchführungsverordnung zum Autonomiegesetz hin, die der Delegation trotz wiederholter Bitten jedoch nicht zur Verfügung gestellt wurde. Der Gouverneur erwähnte überdies, daß eine eigene tibetische Durchführungsverordnung in Ausarbeitung sei.

31. Ein besonderes Kapitel in der chinesischen Staatsstruktur bildet die Stellung sogenannter nationaler Minderheiten. Im Gespräch mit den verantwortlichen Zentralstellen wurde die Andeutung gemacht, daß die Volksrepublik China in der Generalversammlung der Vereinten Nationen der von der UN-Menschenrechtskommission im Wege des ECOSOC vorgelegten Erklärung über die Rechte der Angehörigen nationaler oder ethnischer, sprachlicher und religiöser Minderheiten ihre Zustimmung geben könnte. Die chinesische HanBevölkerung macht laut dem chinesischen Weißbuch für Menschenrechte in Gesamtchina ca. $92 \%$ der Gesamtbevölkerung aus. $8 \%$ fallen "other 55 nationalities" zu. Diese werden in der offiziellen Sprache nicht als "Völker" bezeichnet, sondern als "minority nationalities". Diesen Bevölkerungsgruppen entsprechen 159 nationale autonome Gebiete, von 
denen 5 autonome Regionen sind, zu denen die autonome Provinz Tibet zählt. Den nationalen Minderheiten wird nach chinesischer Auffassung gleiches Recht zuerkannt. Das wird in Art. 4 der chinesischen Verfassung festgelegt.

Es kann in diesem Zusammenhang nicht übersehen werden, daß vor allem die gleichartige wirtschaftliche Entwicklung für alle "minority nationalities" eine Hauptaufgabe darstellt,

32. Es ist offensichtlich chinesische Politik, die Nationalitäten Chinas und auch die Bevölkerung Tibets nicht als "Völker" zu bezeichnen, sondern als Minderheiten. Das muß sich auf die Selbstbestimmungskonzeption, so wie sie durch die Instrumente der UN geordnet wird, auswirken. Auf die Bevölkerungsstruktur Tibets übertragen bedeutet dies, daß die Tibeter zu den "minority nationalities" zählen und so der Minderheitenrechte im chinesischen Staatsverband teilhaftig werden, aber auch den Pflichten unterworfen sind, die die chinesische Verfassung für Minderheiten ausspricht. Der Umstand, daß die chinesische Politik Han-Chinesen im Gebiet Tibets ansässig zu machen strebt, führt nun dazu, daß verschiedene Nationalitäten in Tibet leben, die gleichartige Rechte genießen sollen.

Das 5. Kapitel des Autonomiegesetzes trifft für die Beziehung der Nationalitäten in den autonomen Gebieten besondere Vorschriften. Das bedeutet für Tibet, daß Chinesisch und Tibetisch die of fiziellen Sprachen sind und die Kader die entsprechenden Sprachen in Wort und Schrift lemen sollen. Eine Sprachenzulage wie in Südtirol ist für jene vorgesehen, die zwei oder mehr Sprachen in Wort und Schrift beherrschen, dic in der autonomen Region gesprochen werden (Art. 49 des Autonomiegesetzes). Integration auf dem Gebiet der Wirtschaftsentwicklung, der Entwicklung des Erziehungslebens, des wissenschaftlichen und kulturellen Lebens und der Gesundheitspflege sieht Art. 50 des Autonomiegesetzes vor. Die Organe in der autonomen Provinz sollen im Sinne der Einheit des Landes und der Einheit der Nationalitäten erzogen werden!

Man kann weder den Rechtsvorschriften noch den Gesprächen entnehmen, daß Tibet eine separate Entwicklung zur Wahrung seiner kulturellen und ethnischen Identität erfahren soll. Land und Güter dienen dem ganzen chinesischen Volk (Art. 4 der Verfassung).

\section{V. Über die Menschenrechtsf ragen in Tibet im besonderen}

\section{a) Gewissens- und Religionsfreiheit}

33. Der Gewissens- und Religionsfreiheit kommt angesichts der tiefen Religiosität der Tibeter besondere Bedeutung zu. Sic ist nach chinesischer Auffassung gewährleistet. So ist gemäß Art. 11 des Autonomiegesetzes die Religionsfreiheit, soweit es um die "normalen religiösen Aktivitäten" geht, garantiert. Allerdings erfassen weder die Verfassung noch das Autonomie-(Minderheiten-)Gesetz Religionsangelegenheiten als Gegenstand der Autonomie. Nach Auskunft des Amtes für Religionsangelegenheiten in Peking betrifft die Reli- 
gionsfreiheit die private und kollektive Religionsausübung sowie die religiösen Stätten. Gläubige, Priester oder Ordensleute würden nur verfolgt, wenn sie gegen Gesetze verstoßen. Dies sei auch bei verschiedenen katholischen Bischöfen der Grund gewesen. Es gäbe keine nicht anerkannten Religionsgemeinschaften, auch keine katholische Untergrundkirche, jedoch einen sog. Volksglauben. Religiöse Organisationen hätten eine gewisse Autonomie und die legalen Rechte religiöser Stätten würden gewährleistet. Die von der Delegation neuerlich vorgetragene Idee des österreichischen Außenministers einer Pardonnierung nach neuer gründlicher Prüfung werde an die zuständigen Justizbehörden weitergeleitet werden.

34. Der Staat unterstütze den Wiederaufbau der während der Kulturrevolution zerstörten Tempel und Klöster, von denen nur acht ganz erhalten geblieben seien. Von seiten der UNESCO werde dabei keine Hilfe erhalten. Tatsächlich konnte die Delegation in Tibet an den von ihr besuchten religiösen Stätten eine zum Teil rege Bautätigkeit beobachten. Insbesondere in Potala selbst finden umfangreiche Renovierungsarbeiten statt, die jedoch wohl eher touristisch als religiös motiviert sein dürften. Insgesamt geht der Wiederaufbau der in der Kulturrevolution zerstörten Klöster jedoch schleppend vor sich. Die sog. "normale Religionsausübung" scheint gewährleistet. Geduldet werden auch Bilder des Dalai Lama in allen besuchten Klöstern sowie deren öffentlicher Verkauf am Barkhor.

35. Das Bestehen einer staatlichen Begrenzung der Anzahl der Mönche in den tibetischen Klöstern wurde von offizieller Seite in Abrede gestellt, von allen anderen Quellen jedoch bestätigt. Nach Auskunft der exiltibetischen Seite wird darin jedoch heute ein geringeres Problem erblickt als im Fehlen qualifizierter Lehrer bzw. besserer Ausbildungsmöglichkeiten der jungen Mönche sowie der strikten Kontrolle der Klöster durch chinesische Vertrauensleute und sog. "Arbeitsausschüsse" zur politischen Indoktrination der Mönche. Auch deren Bestehen wurde in Peking in Abrede gestellt. Gegenüber der Delegation des Europäischen Parlaments vom September 1991 war hingegen vom Vorsitzenden der autonomen Region Tibet das Bestehen von acht bis neun politischen Arbeitsteams zugegeben worden, die auf Wunsch von Mönchen und Lamas (!) wegen in den Klöstern bestehender Unabhängigkeitsbestrebungen und darauffolgender Unruhen geschickt worden seien. Die Delegation stellte bei ihrem Besuch eine deutliche Verängstigung der Mönche fest, die aus ihrer allgemeinen Haltung als auch den in einem ad hoc-Gespräch mit dem Vorsitzenden des "Demokratischen Verwaltungskomitecs" im Kloster Ganden erhaltenen Auskünften hervorging. So wurden offensichtlich unwahre Angaben über den Gefängnisaufenthalt von Klostermitgliedern gemacht. Ein unabhängig geführtes Gespräch mit Mönchen war nicht möglich, im Gegenteil, die Delegation sah sich ständig von einer Vielzahl offizieller und anderer Begleiter umgeben. 


\section{b) Anspruch auf rechtliches Gehör - Grundsatz des fair trial}

36. Gemäß Art. 10 der Allgemeinen Erklärung der Menschenrechte hat jeder Mensch hinsichtlich von Entscheidungen über seine Rechte und Pflichten sowie strafrechtliche Beschuldigungen Anspruch auf ein billiges und öffentliches Verfahren vor einem unabhängigen Gericht. Weiters sind nach Art. 11 Para. 1 der Verteidigung im öffentlichen Verfahren alle nötigen Voraussetzungen zu gewährleisten. Diese Mindeststandards, die im Pakt für bürgerliche und politische Rechte sowie in weiteren Instrumenten, denen China nicht angehört, konkretisiert worden sind, erscheinen durch das Justizsystem Chinas nur teilweise erfüllt.

37. Problematisch erscheint insbesondere die späte Einschaltung der Gerichte bei Festnahmen, da die Gerichte normalerweise erst bei der Anklageerhebung befaßt werden, Wenn der Angeklagte keinen Verteidiger, von denen es noch zuwenige gibt, findet oder diesen nicht bezahlen kann, läßt sich das Recht auf Verteidigung nicht verwirklichen. Auch ist die Frist von sieben Tagen vor Prozeßbeginn für die Vorbereitung der Verteidigung in schwierigen Fällen zu kurz. Auffallend ist weiters, daß die allermeisten Angeklagten bzw. ihre Verteidiger auf schuldig plädieren und kaum Freisprüche vorkommen. Die Öffentlichkeit des Verfahrens erscheint durch die kurze Frist der Ankündigung sowie die Ausschlußmöglichkeiten in politisch sensiblen Fällen ebenfalls nicht ausreichend gewährleistet. Schließlich widerspricht v.a. die Einweisung in Umerziehungsanstalten durch nicht-gerichtliche Organe als Sanktion dem Recht auf ein gerichtliches Verfahren, was auch von Seite einzelner chinesischer Juristen anerkannt wird.

\section{c) Freiheitsentzug und Gefängniswesen}

38. Auch wenn im Rechtswesen das Bemühen um Verbesserungen anzuerkennen ist, kann etwa im Hinblick auf den Entzug der Freiheit durch Polizeiorgane und Staatsanwaltschaft ebensowenig von westlichen Standards ausgegangen werden wie hinsichtlich des Gerichtsverfahrens (Grundsatz des fair trail). Jede Betrachtung des Strafvollzuges in China hat davon auszugehen, daß neben den Gefängnissen noch "Anstalten für die Umerziehung durch Arbeit" ("laojiao") bestehen, in denen Personen aufgrund von Beschlüssen von nichtgerichtlichen Organen (Ausschüssen) ohne gerichtliches Verfahren für bis zu vier Jahre festgehalten werden können. Nach einer Broschüre des Justizministeriums der VR China handelt es sich dabei um Zwangsmaßnahmen der Verwaltung für geringere Delikte, die nach dem Strafgesetz nicht strafbar sind. Die Betonung liegt auf Erziehungsmaßnahmen. Da der chinesischen Seite der Mangel an Rechtsstaatlichkeit dieser Form des Freiheitsentzuges zunehmend bewußt wird, ist es schwierig, über derartige Umerziehungslager nähere Informationen oder gar Zugang zu erlangen. In dem im August 1992 veröffentlichten Weißbuch über "Criminal Reform in China" sind diese Institutionen mit keinem Wort 
erwähnt. Da auch in den Strafanstalten aufgrund des Konzepts der Besserung durch Arbeit ("reform through labour" - "laogai") gearbeitet werden muß, kommt es leicht zu Verwechslungen, welche von chinesischen Gesprächspartnern oft bewußt ausgenutzt werden. Sowohl für die Gefängnisse als auch die Umerziehungsanstalten ist das Justizministerium zuständig, wo für letztere ein eigenes Referat besteht. Hinsichtlich der Frage, wie eine Entscheidung über eine gerichtliche Verfolgung oder eine Einweisung in eine Umerziehungsanstalt zustande kommt, war keine klare Antwort zu erhalten.

39. Daneben bestehen auch andere Möglichkeiten der Festhaltung, etwa zwecks Untersuchung eines Falles durch die Sicherheitsbehörden an Orten, die nicht als Gefängnisse deklariert sind, aber auch als solche genutzt werden können und unter der Zuständigkeit der Sicherheitsbehörden stehen. Es gibt verschiedene Formen der Verwaltungshaft, die trotz bestehender Fristen praktisch fast unbeschränkt verlängert werden kann. Sie sind im Bericht der französischen Juristen von 1991 eingehend beschrieben. Nur für die eigentliche Verhaftung bedarf es einer Genehmigung der Justizbehörde (Volksanwaltschaft, $\$ 45 \mathrm{ff}$ Strafprozeßordnung). Eine richterliche Ermächtigung zur Festhaltung benötigen die Sicherheitsbehörden nicht. Auch wäre davon auszugehen, daß dieser mangels einer wirklichen Unabhängigkeit der Gerichte nicht dieselbe Bedeutung wie in einem westlichen Rechtssystem zukommt. Die nach der chinesischen Strafprozeßordnung von 1979 bestehenden Fristen werden nach vorliegenden Berichten auch häufig überschritten. Nach diesen Vorschriften müßten die Familien der Angehörigen oder deren Arbeitseinheit bei Festnahme durch die Polizei innerhalb von 24 Stunden benachrichtigt werden, es sei denn, daß dies die Aufklärung behindern würde (§43 Strafprozeßordnung). Die maximale Polizeihaft im Ermittlungsverfahren beträgt 10 bis 15 Tage (nach in Tibet erhaltenen Informationen 3 bis 7 Tage), nach der eigentlichen Verhaftung mit Genehmigung der Staatsanwaltschaft dürfe die Untersuchungshaft nur in komplizierten Fällen mehr als 3 Monate (nach Aussage in Tibet 1 Monat) dauern etc.

40. Bestätigt wurde, daß die Zuständigkeit für Haftanstalten in Tibet seit Februar 1992 vom Amt für Offentliche Ordnung auf das Justizamt übergegangen ist, was eine Normalisierung darstellt. Die Reaktionen auf bestimmte Fragen lassen allerdings vermuten, daß dieser Ubergang in der Praxis noch nicht ganz vollzogen ist. Darüber hinaus bestehen weitere Orte der Festhaltung, die offensichtlich auch für Sträflinge benutzt werden.

41. Anläßlich des Gefängnisbesuches in Drapchi wurde erklärt, daß Gefangene für schwere Disziplinwidrigkeiten solange, bis sie Selbstkritik übten, max. jedoch 5 Tage in Isolierhaft festgehalten werden können. Dies widerspricht Berichten von ehemaligen Gefangenen nach dem Verlassen Tibets, wonach Gefangene monatelang in Isolierzellen festgehalten würden. Auch andere Informationen, wie daß die Gefangenen den gleichen Lohn und das gleiche Essen wie Arbeitnehmer außerhalb des Gefängnisses erhielten, erscheinen nicht sehr überzeugend. Festzuhalten ist, daß keine gemeinschaftliche Religionsausübung erlaubt ist. 
42. Offensichtlich unrichtig ist weiters die Auskunft, daß Drapchi das einzige Gefängnis in der autonomen Region Tibet sei und daß es auch nur ein Umerziehungslager gäbe. Nach den der Delegation aus verschiedenen Quellen, darunter dem Bericht einer amerikanischen Delegation vom Juli 1991 vorliegenden Informationen bestehen zumindest zwei weitere Gefängnisse, Sangyip und Gutsa, sowie ein weiteres neues Gefängnis, dessen Name noch nicht bekannt ist. Zu Sangyip gehört sowohl ein Strafvollzug durch Arbeit als auch eine Anstalt für die Umerziehung durch Arbeit. Gutsa, das dem städtischen Sicherheitsbüro von Lhasa unterstellt ist, und Sangyip dienen sowohl als Untersuchungsgefängnisse für Vernehmungen als auch für den Strafvollzug, v.a. bei politischen Häftlingen. Sie dürften weiterhin unter der Aufsicht des Amtes für Öffentliche Sicherheit stehen, so daß vielleicht nur Drapchi dem Justizamt unterstellt ist, was die Diskrepanzen zwischen den Aussagen der Behörden und der Flüchtlinge erklären könnte.

\section{d) Folter und menschenunwürdige Behandlung}

43. Hinsichtlich der Frage von Mißhandlungen und Polizeiübergriffen wurde im Gespräch mit dem Justizministerium in Peking zuerst lediglich auf das bestehende gesetzliche Verbot (z.B. § 136 und $\S 189$ des Strafgesetzbuches) hingewiesen und erst auf weiteres Nachfragen 24 schwerere Fälle für ganz China im Jahr 1991 genannt, was auf eine geringe Verfolgungsrate hinweist. Das Weißbuch über "Criminal Reform in China" gibt 24 Verurteilungen von Gefängnispersonal wegen körperlicher Bestrafung von Gefangenen für 1990 und 1991 an. Ebenso zweifelhaft erscheint die Aussage eines Vertreters des Ministeriums für Offentliche Sicherheit, wonach aufgrund der von der Menschenrechtskommission der Vereinten Nationen gemachten Vorwürfe eine Untersuchung eingeleitet worden sei, welche jedoch keinen Anhaltspunkt für die Richtigkeit der Vorwürfe ergeben habe. Das Weißbuch über "Menschenrechte in China" spricht für 1990 von der Einleitung von 472 Gerichtsverfahren wegen Erpressung von Geständnissen durch Folter und Zwang, und in "China Daily" vom 22.7.1992 findet sich ein Bericht, wonach die Volksstaatsanwaltschaften in Hinkunft ihre Aufmerksamkeit stärker auf Fälle illegaler Festnahme sowie Todesfälle und Verletzungen durch Folter während der Untersuchung richten sollen, um die Rechte des Volkes besser zu schützen, womit das Bestehen derartiger Praktiken indirekt zugegeben wird. Auch von seiten der tibetischen Behörden wurden lediglich 20 Fälle von Mißhandlungen bzw. Verstößen gegen die Dienstregeln während der letzten beiden Jahre angegeben, die für die Aufsicht zuständige Staatsanwaltschaft habe nur 10 Beschwerden erhalten. Zugleich wird zugegeben, daß in Tibet nicht alles völlig unter Kontrolle sei. Die Standard Minimum

Rules für Exekutivbeamte seien bekannt. Jedoch müsse bei der Anwendung auf chinesische Gegebenheiten Rücksicht genommen werden.

44. Von ehemaligen Gefangenen wird von zwangsweisen Blutabnahmen berichtet. Durch eine unabhängige Quelle war zu erfahren, daß es sich dabei um kommerzielle Praktiken der 
chinesischen Volksarmee handelt, die das Blut u.a. in Zusammenarbeit mit einer amerikanischen Pharnafirma verwertet. Derartige Praktiken erscheinen mit dem von chinesischer Seite behaupteten Schutz der persönlichen Integrität der Gefangenen nicht vereinbar.

45. Die vielfach behauptete Verwendung von elektrischen Schlagstöcken während der Vernehmung wird geleugnet, da dies nicht zulässig sei. Die Elektronik dürfe nur bei starkem Widerstand (etwa gegen eine Festnahme) eingeschaltet werden. Dem stehen Aussagen von entlassenen Gefangenen gegenüber, wonach in der Untersuchungshaft regelmäßig geprügelt würde sowie andere Foltermethoden zur Anwendung kämen und auch in der Strafhaft Disziplinwidrigkeiten mit harten Prügeln und Isolierhaft geahndet würden. Eine Überprüfung der widersprüchlichen Informationen könnte am besten durch Delegierte des Internationalen Komitees vom Roten Kreuz erfolgen und wäre, wenn die tibetischen Angaben stimmen, auch im Interesse der tibetischen Behörden. Diese gaben auf eine konkrete Frage hin an, noch keinen entsprechenden Antrag erhalten zu haben.

\section{e) Recht auf Bildung}

46. Nach dem Gesetz der Volksrepublik China über die regionale Autonomie der Nationalitäten von 1984 sind vielfältige Maßnahmen vorgesehen, um den Bildungsstand der Minderheitenbevölkerung zu verbessern (Art. 36, 37 und 42). In Schulen, wo die Mehrheit der Studenten der Minderheit angehört, soll in deren Sprache unterrichtet sowie Lehrbücher dieser Sprache verwendet werden (Art. 37). Durch eine Vorzugsbehandlung für die Minderheit soll zumindest Chancengleichheit mit der Mehrheit der Bevölkerung erreicht werden. Schon das Gespräch mit dem Stellvertretenden Vorsitzenden der Kommission des Staatsrates für Nationalitätenangelegenheiten in Peking ergab, daß das Gesetz im Hinblick auf die Verwendung der tibetischen Sprache noch nicht voll durchgeführt worden sei. So können u.a. aufgrund des Lehrermangels auch chinesische Lehrer zum Einsatz kommen, die auf chinesisch unterrichten. Mangels tibetischer Lehrbücher fänden chinesische Bücher Verwendung. An einigen Schulen gäbe es Experimente einer doppelsprachigen Ausbildung.

47. Anläßlich des Besuchs einer Mittelschule in Lhasa war zu erfahren, daß es Klassen in tibetischer, chinesischer und in beiden Sprachen (Tibetisch als Unterrichts- und Chinesisch als Schriftsprache) gebe. Kinder, die die tibetischen Klassen besuchen, haben erst später Englischunterricht, da sie erst Chinesisch lernen müssen. Hinsichtlich der Aufnahme an die Universität wären trotz geringerer Anforderungen für tibetische Studenten 35-40\% der Aufgenommenen Han-Chinesen gewesen. Nur einige der chinesischen Lehrer könnten auch Tibetisch. Von den 10 Englischlehrern der Schule waren nur drei Tibeter. Positive Maßnahmen zur Förderung der tibetischen Schüler, die allgemein ein schwächeres Niveau aufwiesen, seien versucht, jedoch mangels Erfolg nicht fortgeführt worden. Klagen über die 
Qualität des Unterrichts in tibetischer Sprache gegenüber den Klassen in chinesischer Sprache sind der Kommission bekannt, konnten jedoch vor Ort nicht überprüft werden. So wird allgemein eine Vernachlässigung des Bildungswesens auf dem Land gegenüber den Städten und eine schlechtere Ausstattung der tibetischen Klassen kritisiert.

48. Auf dem Flug von und nach Chengdu traf die Delegation auf Schüler- und Studentengruppen, die von China nach Hause zurückkehrten. Eine große Anzahl von Schülem wird in verschiedenen Provinzen Chinas ausgebildet und kommt z.B. in der Oberstufe der Mittelschule bis zu vier Jahre nicht nach Hause, was trotz Tibetisch-Unterrichts ein großes Problem der Entfremdung aufwirft. Es stellt sich die Frage, warum die für die Ausbildung in China eingesetzten beträchtlichen Ressourcen nicht für eine Verbesserung der Ausbildung in Tibet verwendet werden.

49. Anläßlich des Besuchs der hauptsächlichen Universität von Tibet zeigte sich, daß die Autonomie der Universität sowie der autonomen Region relativ beschränkt sind. So würde der Großteil der Lehrkräfte von der Zentralregierung zugeteilt. Etwa 300 Tibeter und 150 Han-Chinesen (das sind $33 \%$ ) bestehen jährlich die Aufnahmeprüfung für Hochschulen in anderen Provinzen. Es kann daraus geschlossen werden, daß das Bildungssystem in Tibet es den Tibetern nicht erlaubt, eine gleiche Qualifikation wie die Han-Chinesen zu erreichen, was in einem überproportionalen Anteil der Han-Chinesen beim Zugang zum Hochschulstudium zum Ausdruck kommt.

50. Besonders krass ist das Mißverhältnis hinsichtlich der Studienmöglichkeiten im Ausland. Schon in Peking war nur von wenigen tibetischen Studenten die Rede, die im Ausland studierten, in Lhasa waren aus den beiden letzten Jahren trotz ca. 3000 Regierungsstipendien pro Jahr nur zwei derartige Fälle bekannt. Dies scheint weniger an den Stipendienmöglichkeiten zu liegen als an der notwendigen Zustimmung der staatlichen Erziehungskommission. Schon aus dem Jahr 1987 sind Klagen bekannt, wonach z.B. nur vier tibetische Studenten in den USA studieren konnten. Daran scheint sich jedoch bis heute nichts Grundlegendes geändert zu haben.

\section{f) Recht auf Arbeit}

51. Nach chinesischer Darstellung hat jeder Arbeitsfähige grundsätzlich ein Recht auf Arbeit. Gewerkschaften bestehen in Tibet jedoch nur für Angestellte und Betriebsarbeiter, nicht für Landarbeiter und Bauem, die zusammen mit den Nomaden etwa 80 \% der Bevölkerung ausmachen. Die Bauern sollen bis mindestens 1994 Steuerf reiheit genießen. Genossenschaften gibt es nicht. Die Bauem haben völlige Freiheit hinsichtlich des Verkaufs ihrer Produkte. Die Han-Chinesen betrieben keine Landwirtschaft; allerdings hätten einige Böden gepachtet, um darauf Gemüse anzubauen. 


\section{g) Recht auf Umwelt}

52. Sowohl von seiten intemationaler Umweltorganisationen als auch der tibetischen Exilregierung wurde wiederholt auf die Gefahren einer Schädigung der fragilen Ökologie des tibetischen Hochlandes aufmerksam gemacht und auf den Kahlschlag von Wäldem, v.a. in Osttibet, hingewiesen. Nach Aussage des Gouverneurs von Tibet werden in der autonomen Region regelmäßig Wiederaufforstungsprogramme durchgeführt. Hinsichtlich der Tätigkeit chinesischer Bergbauunternehmen auf tibetischem Gebiet blieb der Eindruck einer nur begrenzten Kontrollmöglichkeit.

53. Näher erörtert wurde das Projekt eines Pumpspeicherkraftwerkes (Yang Zhuo Yong), das einen den Tibetern heiligen See nutzen soll und dessen Maschinenausstattung von der österreichischen Firma Elin geliefert wird. Nach Angaben des Gouverneurs hat sich die Bevölkerung nach ursprünglichem Widerstand von der Regierung überzeugen lassen. Mittels geothermischer Energie werde das dem See entnommene Wasser großteils wieder zurückgepumpt, so daß der Wasserspiegel konstant bleibe. Die Firma Elin bestätigte dies, stützt sich dabei jedoch nur auf chinesische Berechnungen. Im Ausland wird das Projekt aus kulturellen und ökologischen Gründen kritisiert. Der Vorstandsvorsitzende der Elin AG wird das von chinesischer Seite bereits weitgehend fertiggestellte Projekt im Oktober selbst besuchen. Es wäre wichtig dafür Sorge zu tragen, daß die erhaltenen Angaben überprüft werden, damit der Ruf Österreichs nicht durch ein chinesisches Regierungsprojekt in Mitleidenschaft gezogen wird. Nach Angaben eines im Rahmen eines deutschen Entwicklungsprojekts tätigen österreichischen Experten wären für die Tibeter v.a. dezentrale Kleinkraftwerke zur Nutzung der Wasserkraft von Vorteil.

\section{h) Rechtsstaatlichkeit und Menschenrechte}

54. China ist ein Entwicklungsland. Dies gilt für das Rechtswesen ebenso wie für den Schutz der Menschenrechte. So wurde eine westlichem System vergleichbare Rechtsordnung erst in der Zeit nach der Kulturrevolution entwickelt, und noch bestehen bedeutende Lücken und Unzulänglichkeiten. In seiner langen Geschichte wurde China geprägt durch seinen Zentralismus und die Vorherrschaft der Exekutive. Eine Gewaltenteilung im liberalen Sinne begann sich erst in jüngerer Zeit zu entwickeln, kann jedoch ebenso wie die Herrschaft des Rechts (rule of law) noch nicht als allgemein wirksam angesehen werden. Die eigentliche Steuerung des sozialen Verhaltens verläuft vielfach über dem auswärtigen Beobachter nicht zugänglichen Entscheidungsmechanismen, wodurch die Bedeutung der formalen Institutionen und Rechtsvorschriften relativiert wird. Die Rechtsstaatlichkeit wird ferner durch das große Ermessen bei der Beurteilung von Tatbeständen, wie der "konterrevolutionären Straftaten", worunter alle Aktivitäten zum Sturz der Macht des Proletariats und der Gefährdung des sozialen Systems verstanden werden, oder durch weite Ausnahme- 
bestimmungen bzw. Pflichten relativiert. So dürfen die Bürger gemäß dem Weißbuch über Menschenrechte bei der Ausübung ihrer politischen Rechte die Interessen des Staates, der Gesellschaft oder des Kollektivs sowie anderer Bürger nicht verletzen. Eigenständige Bemühungen um eine größere Rechtsstaatlichkeit beruhen vor allem auf der Erfahrung der Kulturrevolution und der Notwendigkeit einer höheren Stabilität als Rahmenbedingung einer rascheren Entwicklung. Positiv ist auch zu vermerken, daß kaum versucht wurde, diese Unzulänglichkeiten unter Berufung auf eine andere Menschenrechtskonzeption zu rechtfertigen. Ebenso wurde um Verständnis ersucht und der Wille ausgedrückt, Verbesserungen durchführen zu wollen, wobei man am Gespräch mit dem Ausland interessiert sei. Es bleibt zu hoffen, daß die Entwicklung zu größerer Rechtsstaatlichkeit auch der Situation der Menschenrechte in Tibet zugute kommen wird.

\section{Schlußfolgerungen und Empfehlungen}

\section{a) Schlußfolgerungen}

Die österreichische Rechtsexpertendelegation kommt nach dem Besuch Chinas bzw. Tibets im Juli 1992 und dem Studium der einschlägigen Dokumente zu den nachstehenden Schlußfolgerungen:

1. China ist durch die Reaktion der Weltöffentlichkeit auf die blutige Niederschlagung der Volksaufstände in Lhasa von 1987, 1988 und 1989 bemüht, eine Politik der Rekonziliation zu führen und zu demonstrieren.

2. Dabei wird das Unrecht anerkannt, das im Zuge der Kulturrevolution Tibet und seinem Volke zugefügt worden ist.

3. Nach Völkerrecht steht dem tibetischen Volke das Recht auf Selbstbestimmung zu; es erfüllt alle Bedingungen, die das Völkerrecht an die Trägerschaft des Selbstbestimmungsrechtes stellt. Die Tibeter im Exil verlangen vor allem Unabhängigkeit - eines der Modelle des Selbstbestimmungsrechts.

4. Die Volksrepublik China ist jedoch nicht gewillt, der Unabhängigkeit Tibets und damit der Ausübung dieser Form des Selbstbestimmungsrechts des tibetischen Volkes näherzutreten. Sie beruft sich u.a. auf eine Abmachung mit Abgesandten des 14. Dalai Lama von 1951 über das Schicksal Tibets, eine Abmachung, die als eine Art Unterwerfung Tibets unter die Souveränität Chinas gedeutet worden ist.

5. Obwohl im Zuge der Rekonziliationspolitik deutliche Zeichen im Bemühen Chinas bestehen, die Auswüchse der Kulturrevolution, die Tibet betroffen haben wettzumachen, ist 
die fortschreitende personelle, wirtschaftliche und politische Integration Tibets in die Volksrepublik nicht zu übersehen.

6. Ansätze für eine fortschreitende Verdrängung des tibetischen Volkes durch Veränderungen der demographischen Struktur Tibets sind erkennbar. Eine völlige Assimilierung der Tibeter scheint allerdings unwahrscheinlich, weil eine Bereitschaft der Han-Chinesen, aus freien Stücken in Tibet zu siedeln, sehr gering ist. Jedoch ist eine zunehmende Beherrschung des Wirtschaftslebens durch diese festzustellen.

7. Die chinesische Nutzung der Naturschätze Tibets primär für Zwecke der Volksrepublik ist unverkennbar. Auch dies steht nicht im Einklang mit den einschlägigen menschenrechtlichen Normen, wie sie insbesondere in den Menschenrechts-Pakten enthalten sind.

8. Tibet hat im Rahmen einer nach marxistisch-leninistischen Grundsätzen konstruierten Staatsstruktur eine Selbstverwaltung als nationale Autonomie.

9. Die autonome Region Tibet (TAR) hat eine Art Gesetzgebungs- und Verwaltungsautonomie. Die autonomen Bereiche beziehen sich vor allem auf die Erziehung, die Landeskultur, die örtliche Sicherheit und die wirtschaftliche sowie soziale Entwicklung. Die Ausübung der Autonomie unterliegt jedoch dem demokratischen Zentralismus und dem kommunistischen Organisationsprinzip der sogenannten doppelten Unterstellung. Die Autonomie ist nicht dazu bestimmt, die besonderen Charakteristika des tibetischen Volkes weiterzuentwickeln oder zur weiteren Entfaltung zu bringen. Der tibetischen Religion wird daher keine Sonderstellung als die Nationalität tragender Faktor eingeräumt - im Gegenteil. Die Trennung von Staat und Religion, die die Verfassung verfügt, war vor allem wegen der Ereignisse der Kulturrevolution als eine religionsfeindliche Trennung anzusehen. Heute hat die Trennung einen die Religion beeinträchtigenden Charakter.

10. Die Gerichtsbarkeit fällt nicht in den autonomen Bereich, sie ist in die gesamtchinesische Hierarchie, abgesehen von personellen Zusammensetzungen der Gerichte und der Staatsanwaltschaft, eingegliedert.

11. Der den Nationalitäten eingeräumte Minderheitenschutz in Tibet nimmt nicht auf die besondere Verknüpfung der tibetischen Nationalität mit der buddhistischen Religion Bedacht. Eine Gleichstellung der nationalen Minderheit wird durch das ausschließlich ethnische Konzept des Minderheitenschutzes herbeigeführt. Es kennt die Kombination der Charakteristika des Nationalen und Religiösen nicht.

12. Das chinesische Nationalitätenkonzept erzeugt für Tibet einen Wertekonflikt zwischen angestammter und ausgeübter Religiosität und fortschrittsgläubiger Pragmatik. 
13. Die Klosterbesuche zeigen, daß das Bildnis des 14. Dalai Lama überall sichtbar ist. Diese Bekundung ist Ausdruck der allgemeinen Verehrung, die der Dalai Lama in Tibet genießt.

Der Wiederaufbau der in der Kulturrevolution zerstörten Klöster geht teilweise mit staatlicher Unterstützung schleppend vor sich. Das Kloster Ganden ist ein diesbezüglich markantes Beispiel.

Gerade dort ist die Dezimierung der Mönche augenscheinlich. Sei es, daß die Mönche während der Kulturrevolution geflohen sind, sei es, daß sie durch staatliche Zwangsmaßnahmen an weitgehend unbekannten Bestimmungsorten festgehalten werden, sei es, daß sie andere Berufe ergriffen haben. Der Nachwuchs der Mönche, vor allem der den Dialog praktizierenden und lehrenden Erzieher wird durch staatliche Maßnahmen erschwert. Das Fehlen qualifizierter Lehrer stellt ein Hauptproblem in den Klöstern dar.

Jedes Kloster muß ein politisches Mönchskomitee ("demokratisches Komitee") haben, das die Verbindung zwischen Kloster und Obrigkeit herzustellen hat. Die wirkliche Obrigkeit ist die Partei. Diese Komitees repräsentieren nicht das Mönchstum in Tibet an sich, sondern nur das jeweilige Kloster. Die Klöster werden streng kontrolliert. So hielt sich während der letzten vier Monate vor unserem Besuch ein sog. "work committee" im Kloster Drepung auf, um die Mönche zu überprüfen und ideologische Erziehungsarbeit zu leisten. Das Bestehen von Höchstgrenzen für die Aufnahme von Mönchen in die Klöster wird von Regierungsseite nicht zugegeben, ist jedoch allgemein bekannt. Derzeit werden diese Beschränkungen jedoch teilweise zurückhaltend gehandhabt.

14. Die Religionsausübung scheint in den Klöstern und auf den Pilgerpfaden relativ ungehindert zu sein. Allerdings dürfen bestimmte Gebete nicht gesprochen werden und polizeiliche Zivilstreifen beobachten die Religionsausübung. Die private Religionsausübung in der Familie scheint gewährleistet, öffentliche Aufgaben dürfen von den Religionsgemeinschaften in der Regel jedoch nicht ausgeübt werden.

15. Die Menschenrechtslage in Tibet ist unter den geschilderten Rahmenbedingungen zu verstehen. Eine politische Meinungsäußerung ist nur im Rahmen und auf der Grundlage der herrschenden Machtverhältnisse möglich. Andere politische Meinungen werden mit Härte unterdrückt. Ansonsten scheint vor allem für politisch nicht interessierte Besucher der Zustand relativ normal zu sein. Im Kontakt mit Fremden herrscht seitens der tibetischen Bevölkerung jedoch offensichtlich Angst, sobald politische Themen aufgeworfen werden. Die Regierung stellt die wirtschaftlichen Rechte und ihre Bemühungen, die Lebensbedingungen der Tibeter zu verbessern, in den Vordergrund.

16. $\mathrm{Zu}$ unterstreichen ist, daß sich die allgemeine Menschenrechtssituation in Tibet nicht wesentlich von der Situation in China im allgemeinen unterscheidet, jedoch wegen der als Staatsfeind Nr. 1 betrachteten Unabhängigkeitsbewegung größere Härte gezeigt wird. Anders als in China selbst gibt es jedoch keine Demokratiebewegung. Die gegenwärtige 
Politik der wirtschaftlichen Öfnung steht somit im Gegensatz zur Härte des Vorgehens gegen politisch Andersdenkende. Dies gilt für ganz China, besonders jedoch für Tibet. Eine Politik der Versöhnung mit dem politischen Gegner scheint zumindest offiziell nicht existent.

17. Übergriffe gegen Untersuchungshäftlinge oder Strafgefangene, Folter zur Erzwingung von Geständnissen oder langandauernde Isolierhaft werden als verboten in Abrede gestellt. Jedes Eintreten für die Unabhängigkeit Tibets wird rigoros verfolgt und bestraft, auch gewaltfreie Demonstrationen, etwa im Form des Vorzeigens einer tibetischen Flagge, begründen bereits ein konterrevolutionäres Vergehen. Derartige Personen, derzeit handelt es sich meist um Mönche und Nonnen aus umliegenden Klöstern, gelten als Separatisten und werden meist für zwei bis drei Jahre in Anstalten zur Umerziehung durch Arbeit geschickt oder zu Gefängnisstrafen verurteilt, worauf sie nicht mehr in ihre Klöster zurückkehren dürfen.

18. Das von der Delegation besuchte Gefängnis Drapchi in Lhasa wurde erst im Februar 1992 der Zuständigkeit des Justizministeriums unterstellt. Zuvor war das Ministerium für Offentliche Sicherheit zuständig gewesen. Weitere, auch als Gefängnisort genützte Festhaltungsorte scheinen weiterhin unter der Aufsicht des Amtes für Öffentliche Sicherheit zu stehen.

19. Hinsichtlich des Rechts auf Bildung scheinen die Tibeter trotz anerkennenswerter Anstrengungen faktisch weiterhin benachteiligt, da der Unterricht in tibetischer Sprache zu Lasten der höheren Bildungsmöglichkeiten geht und eigene Stützungskurse für tibetische Kinder nicht existieren. Auch gibt es z.B. für tibetische Studenten so gut wie keine Möglichkeit zu Auslandsstudien.

20. Die tibetischen Behörden sind nicht bereit, den Zuzug der Han-Chinesen irgendwelchen Beschränkungen zu unterwerfen, obwohl die Möglichkeit einer Kontrolle in Art. 43 des Minderheitengesetzes von 1984 grundsätzlich vorgesehen ist. Dazu kommt die Gefahr der Verdrängung der tibetischen Kultur durch die wachsende Präsenz des chinesischen Fernsehens. Zwar wird auch das Fernsehen in tibetischer Sprache ausgebaut, doch besteht seit 1991 aufgrund einer neuen Satellitenübertragungsanlage erstmals die Möglichkeit, die chinesichen Fernsehprogramme in ganz Tibet in vollständiger Form auszustrahlen.

\section{b) Empfehlungen}

1. Osterreich sollte darauf hinwirken, daß die Volksrepublik China den Menschenrechtspakten der Vereinten Nationen beitritt und auch das Fakultativprotokoll zum Pakt über die zivilen und politischen Rechte unterzeichnet. 
2. Jede bewußte demographische Änderung in Tibet soll hintangehalten werden.

3. Die Tibeter sollen auch als "religiöse Minderheit" geschützt werden.

4. Ein besonderer Ausgleich von wirtschaftlichem Fortschritt und Umweltschutz unter Berücksichtigung religöser Belange und Interessen sollte angestrebt werden.

5. Am Wiederaufbau der während der Kulturrevolution zerstörten Klöster sollte verstärkt mitgewirkt und dafür die Unterstützung der UNESCO gesucht werden.

6. Die bedingungslose Rückkehr der tibetischen Exilanten in Freiheit und Würde soll ermöglicht werden.

7. Die chinesischen Behörden sollten eine unabhängige Überprüfung aller tibetischen Haftanstalten zulassen; dem Internationalen Komitee vom Roten Kreuz (ICRC) sollte ungehinderter Zutritt zu allen diesbezüglichen Haftanstalten ermöglicht werden.

8. Die vom ECOSOC beschlossenen "Standard Minimum Rules for the Treatment of Prisoners" sollten Eingang in das chinesische Strafvollzugsrecht finden.

9. Die österreichische Bundesregierung sollte in der Abwicklung der Stipendienprogramme für Angehörige von Entwicklungsländern eine Mindestanzahl von Stipendien für tibetische Studenten reservieren und auch auf deren Inanspruchnahme als Teil des Gesamtprogrammes drängen.

10. Die Öffnung Tibets und die neue Kooperationsbereitschaft der Regierung der Autonomen Region Tibet sollte für Projekte wirtschaftlicher als auch der Entwicklungskooperation im Sinne positiver Maßnahmen genutzt werden. Diese Projekte und Aktivitäten sollten vor allem der tibetischen Bevölkerung zugute kommen. Bei allen Projekten, vor allem im Bereich der Energieversorgung (Wasserkraft) und des Tourismus, sollte auch auf den Umwelt- und Kulturgüterschutz in Tibet Bedacht genommen werden.

11. Um zukünftigen in- und ausländischen Delegationen, die China und Tibet besuchen, diesen Besuch zu erleichtern, sollte dieser Bericht der österreichischen Expertendelegation einer Veröffentlichung zugeführt werden.

12. Ein Problem des Verständnisses des chinesischen Rechtssystems liegt in der Existenz von Institutionen vor allem im Verwaltungsbereich mit weitreichenden Kompetenzen, die in den im Westen bekannten Institutionen keine Entsprechung finden. Unter demselben Namen bestehen oft sehr unterschiedliche Einrichtungen, die unter ganz anderen Rahmenbedingungen funktionieren. Den chinesischen Gesprächspartnern fehlt vielfach das Verständnis für die Bestimmungsfaktoren und Rahmenbedingungen westlicher Rechtsstaatlichkeit, so daß der Fortsetzung des Dialogs große Bedeutung zukommt. 


\title{
ABSTRACTS
}

\author{
The Sovereignty of States and the New Role of the United Nations Security Council
}

\section{By Jost Delbrück}

The first part explains the international lex lata concerning the protection of state sovereignty and the legal restrictions to which sovereignty has been subjected over the last several decades. The main focus of this part is the recognition of sovereignty as a basic principle of the international community within and outside of the United Nations (art. 2(2) UN Charter) and the meaning and scope of the prohibition of interventions by the UN into the domestic affairs of member states under art. 2(7) UN Charter. The author concludes that in view of the internationalization of the protection of human rights, the principle of nonintervention has been increasingly narrowly construed by the Security Council and the General Assembly. The second part is devoted to a description of future international and domestic conflict scenarios, primarily involving grave human rights violations, likely to demand international action.The third part outlines the means of action available to the organized international community and tries to indicate the legal criteria for and limitations on lawful intervention by the international community in the type of scenarios described. The author concludes that the Security Council should play a more active role and that, at the same time, international law provides for sufficient legal restraints on Security Council actions in order to maintain adequate protection for the sovereignty of states affected by such actions.

\section{Report of the Austrian Legal Fact-Finding Delegation to China/Tibet in July 1992}

\section{By Felix Ermacora and Wolfgang Benedek}

In July 1992, an Austrian delegation of legal experts was given the opportunity to visit Peking and Lhasa, Tibet, to inquire into the situation of human rights in Tibet and to study the relevant legal system of China. It was the only delegation of this kind in 1992. The report by Ermacora and Benedek, after giving a historical account of the Tibetan question, deals with the general aspects of human rights in the Tibetan issue, the question of independence of Tibet and the treatment of Tibetans as a minority nationality including the issue of autonomy as well as a number of human rights like freedom of religion, fair trial and right to education. The relationship between the rule of law and human rights in China 
is also discussed. The report ends with a number of conclusions of mainly legal character and some recommendations the delegation has addressed to the Austrian government, which, however, may also be of general relevance.

\section{The Economic Constitution of Taiwan - The Situation at the Turning Point}

\section{By Wolfgang Lasars}

Taiwan, that is the Republic of China, understands herself as the anti-communist representative of a free China, on the other hand liberals in Taiwan demand a fundamental marketoriented reform of the Economic Constitution. To understand this contradiction the article examines the Economic Constitution exemplified by the development and the extent of the freedom of enterprises in Taiwan.

On one hand the system of private property and the freedom of enterprises are guaranteed by the Constitution. On the other hand emergency laws which go back to World War II and the civil war against the Chinese Communists have restricted the freedom of enterprises for several decades until 1992. The freedom of enterprises is even more curbed by a large sector of public enterprises and a special sector of corporations owned directly by the ruling party KMT. Because of the dominance of the privileged corporations of the state and the party KMT in the domestic market, many small and medium sized companies have to turn to the export sector. Another related aspect of the Economic Constitution of Taiwan is a large area of illegal but tolerated economic activity. From the beginning onwards, the liberal and democratic opposition in Taiwan has stringly criticized this Economic constitution imposed by the KMT.

Now Taiwan has reached a tuming point: Most probably, the emergency laws will be scrapped, concerning the public corporations the government has initialled first steps in the direction of privatization. However, on the other hand, the corporate sector of the ruling party KMT is still growing. It remains to be seen to which results the present reform process of the Economic Constitution finally will lead.

Mongolia on the Way to Pluralistic Democracy and Market Economy - The New Mongolian Constitution of 1992

\section{By Jürgen Merz}

The communist breakdown in the USSR lead to significant political and constitutional changes in Mongolia as well and initiated a period of transition from totalitarianism and 